TRANSACTIONS OF THE

AMERICAN MATHEMATICAL SOCIETY

Volume 358, Number 2, Pages 657-670

S 0002-9947(05)03664-0

Article electronically published on February 4, 2005

\title{
NEWTON POLYGONS AND LOCAL INTEGRABILITY OF NEGATIVE POWERS OF SMOOTH FUNCTIONS IN THE PLANE
}

\author{
MICHAEL GREENBLATT
}

\begin{abstract}
Let $f(x, y)$ be any smooth real-valued function with $f(0,0)=0$. For a sufficiently small neighborhood $U$ of the origin, we study the number

$$
\sup \left\{\epsilon: \int_{U}|f(x, y)|^{-\epsilon}<\infty\right\} \text {. }
$$

It is known that sometimes this number can be expressed in a natural way using the Newton polygon of $f$. We provide necessary and sufficient conditions for this Newton polygon characterization to hold. The behavior of the integral at the supremal $\epsilon$ is also analyzed.
\end{abstract}

\section{INTRODUCTION}

If $p(x)$ is a smooth real-valued function defined on an interval containing 0 , then the set of positive $\epsilon$ for which $\int_{I}|p(x)|^{-\epsilon}$ is finite for all sufficiently small intervals $I$ containing 0 is given by $\left(0, \frac{1}{k}\right)$, where $k$ is the order of the zero of $p$ at 0 . One might ask the analogous question in two variables, namely, if $p(x, y)$ is a smooth function defined on a neighborhood of $(0,0)$, for which positive $\epsilon$ is it true that for a sufficiently small neighborhood $U$ of the origin we have

$$
\int_{U}|p(x, y)|^{-\epsilon}<\infty
$$

For real-analytic functions, this question was considered in [PSSt, and weighted generalizations are analyzed in $[\mathrm{P}$. There is also a direct relation between this question and oscillatory integrals of the type considered in $[\mathrm{V}]$, but this will not be discussed here. By Taylor expanding $p(x, y)$ at the origin, it is not hard to see that in order for there to be any positive $\epsilon$ at all satisfying (1.1), there must be a multi-index $(a, b)$ with $\partial_{x}^{a} \partial_{y}^{b} p(0,0) \neq 0$. In other words, $p$ must be of finite-type. In algebraic geometry, the local behavior of zeroes of finite-type functions such as polynomials are often analyzed with the use of the notion of the Newton polygon of a function, and this concept is useful here as well. In such arguments, it is generally prudent to assume that $p(x, y)$ is smooth. If we stipulate less regularity, for example, if we only require that one mixed partial $\partial_{x}^{a} \partial_{y}^{b} p(0,0)$ exists and is nonzero, then analyzing (1.1) would be much harder; we refer the reader to [CCW]

Received by the editors November 11, 2002 and, in revised form, February 6, 2004

2000 Mathematics Subject Classification. Primary 42B20; Secondary 35H20.

Key words and phrases. Resolution of singularities, Newton polygon.

This research was supported in part by NSF grant DMS-9988798. 
for a combinatorial approach that has yielded some information in such a more general situation.

We now give some definitions. Suppose $p(x, y)$ is a smooth finite-type function in a neighborhood of the origin with (possibly nonconvergent) Taylor expansion $\sum_{m, n} a_{m, n} x^{m} y^{n}$. For each $(m, n)$ in $\mathbf{R}^{2}$, let $Q_{m, n}$ be the set $\left\{(x, y) \in \mathbf{R}^{2}: x \geq\right.$ $m, y \geq n\}$.

Definition. The Newton polygon $N(p)$ of $p$ is defined to be the convex hull of all of the $Q_{m, n}$ for which $a_{m, n} \neq 0$.

Definition. The Newton distance $N D(p)$ of $p$ is defined to be the least $\delta>0$ such that $(\delta, \delta) \in N(p)$.

Geometrically, $N D(p)$ is the $x$-coordinate of the intersection of the line $y=x$ with $N(p)$.

It is easy to see that $N(p)$ must have finitely many extreme points. As a result, the boundary of $N(p)$ consists of an infinite vertical ray, an infinite horizontal ray, and finitely many (possibly zero) slanted segments between the two rays, the slopes of which get larger (less negative) as one goes counterclockwise from the vertical ray to the horizontal ray of the polygon.

To see how Newton polygons are of use in the problems at hand, suppose for now that $p(x, y)=\sum_{m, n} p_{m, n} x^{m} y^{n}$ is a polynomial with positive coefficients, such that $p(0,0)=0$ and only terms with even powers of both $x$ and $y$ are nonzero. This ensures that the zero set of $p$ is only the origin and that $p$ grows in such a way that the Newton polygon is readily applicable. We consider the behavior of $p$ in the quadrant $\{x>0, y>0\}$, the other three being analogous. Every point $(x, y)$ in the upper right quadrant with $y<1$ can be written as $\left(x, x^{d}\right)$ for some $d>0$, so to analyze the local behavior of $p$ in this quadrant it helps to look at the behavior of $p$ along various curves $C_{d}$, where $C_{d}$ is defined for a fixed but small $x_{0}$ by

$$
C_{d}=\left\{\left(x, x^{d}\right): 0<x<x_{0}\right\} .
$$

Observe that we have

$$
p\left(x, x^{d}\right)=\sum_{m, n} p_{m, n} x^{m+d n} .
$$

So since all the coefficients are positive, if $x_{0}$ is sufficiently small we have on $C_{d}$ that

$$
p\left(x, x^{d}\right) \sim \sup _{\left\{(m, n): p_{m, n} \neq 0\right\}} x^{m+d n}=x^{e} .
$$

Here $e$ is defined by

$$
e=\inf _{\left\{(m, n): p_{m, n} \neq 0\right\}} m+d n .
$$

Thus $e$ can be interpreted as the $x$-intercept of the lowest line of slope $-\frac{1}{d}$ intersecting the Newton polygon $N(p)$ of $p$. If $v$ is a vertex of $N(p)$, between edges of slopes $-\frac{1}{m}$ and $-\frac{1}{m^{\prime}}$, then this lowest line will intersect $N(p)$ at $v$ whenever $m \leq d \leq m^{\prime}$. The natural analogues also hold when $m=0$ and/or $m^{\prime}=\infty$. Hence if we denote the slopes of the edges of $N(p)$ in increasing order by $-\frac{1}{m_{0}}, \ldots,-\frac{1}{m_{k}}$, and the vertex between the $m_{i}$ edge and the $m_{i+1}$ edge by $\left(a_{i}, b_{i}\right)$, then when $m_{i} \leq d \leq m_{i+1}$ we heuristically have

$$
p\left(x, x^{d}\right) \sim x^{a_{i}+d b_{i}} .
$$


This is only heuristic since we have not done the additional work that shows that the $x_{0}$ in (1.2) and subsequent equations can be taken uniformly in $d$. Ignoring this, we define the region $D_{i}$ by

$$
D_{i}=\left\{(x, y): 0<x<x_{0}, x^{m_{i+1}}<y<x^{m_{i}}\right\} .
$$

We can now analyze the integral (1.1). Namely, given $\epsilon>0$ and any $0<y_{0}<1$, we have that

$$
\begin{gathered}
\int_{\left\{x_{0}>x>0, y_{0}>y>0\right\}} p(x, y)^{-\epsilon} \\
<\int_{\left\{x_{0}>x>0,1>y>0\right\}} p(x, y)^{-\epsilon}=\sum_{i} \int_{D_{i}} p(x, y)^{-\epsilon} \\
\sim \sum_{i} \int_{D_{i}} x^{-\epsilon a_{i}} y^{-\epsilon b_{i}} .
\end{gathered}
$$

We perform the integration in each term of (1.5), first with respect to $y$ and then with respect to $x$. Doing the $y$-integration for the $i$ th term we obtain a quantity $I_{i}^{x}$ as follows:

Case (1). If $\epsilon<\frac{1}{b_{i}}$, then $I_{i}^{x} \sim x^{m_{i}\left(-b_{i} \epsilon+1\right)-a_{i} \epsilon}$, and $\int_{0}^{x_{0}} I_{i}^{x}<\infty$ iff $\epsilon<\frac{1+m_{i}}{a_{i}+m_{i} b_{i}}$.

Case (2). If $\epsilon>\frac{1}{b_{i}}$, then $I_{i}^{x} \sim x^{m_{i+1}\left(-b_{i} \epsilon+1\right)-a_{i} \epsilon}$, and $\int_{0}^{x_{0}} I_{i}^{x}<\infty$ iff $\epsilon<\frac{1+m_{i+1}}{a_{i}+m_{i+1} b_{i}}$. Note that when this integral is finite we have $\frac{1}{b_{i}}<\epsilon<\frac{1+m_{i+1}}{a_{i}+m_{i+1} b_{i}}$ and thus $b_{i}>a_{i}$.

Case (3). If $\epsilon=\frac{1}{b_{i}}$, then $I_{i}^{x} \sim x^{-a_{i} \epsilon} \ln x=x^{m_{i}\left(-b_{i} \epsilon+1\right)-a_{i} \epsilon} \ln x$, and $\int_{0}^{x_{0}} I_{i}^{x}<\infty$ iff $\epsilon<\frac{1+m_{i}}{a_{i}+m_{i} b_{i}}$. As in case (2), if the integral is finite we must have $\frac{1}{b_{i}}=\epsilon<\frac{1+m_{i}}{a_{i}+m_{i} b_{i}}$ and hence $b_{i}>a_{i}$.

Geometrically, the number $\frac{1+m_{i}}{a_{i}+m_{i} b_{i}}$ is the reciprocal of the $x$-coordinate of the intersection of the line $y=x$ with the line containing the edge of $N(p)$ of slope $-\frac{1}{m_{i}}$. Hence the quantity in (1.5) is finite if $\epsilon$ is less than $\frac{1+m_{i}}{a_{i}+m_{i} b_{i}}$ for every $m_{i}$ corresponding to an edge of $N(p)$. Equivalently, taking the minimum of these $m_{i}$, if we denote the slope of the edge of $N(p)$ intersecting the line $y=x$ by $-\frac{1}{\tilde{m}}$, we have the following sufficient condition of finiteness of (1.5):

$$
\epsilon<\frac{1+\tilde{m}}{a_{i}+\tilde{m} b_{i}}=\frac{1}{N D(p)}
$$

The condition (1.6) is necessary as well. Let $\left(a_{i}, b_{i}\right)$ be the vertex of $N(p)$ with $b_{i} \leq a_{i}$ such that $b_{i}$ is maximal. (If such a vertex doesn't exist, switch the roles of the $x$ and $y$ axes.) Since $b_{i} \leq a_{i}$, as observed above, for the term of (1.5) corresponding to $\left(a_{i}, b_{i}\right)$ to be finite, we must be in Case 1 . In this situation $m_{i}=\tilde{m}$ and therefore if the term is finite, then (1.6) must hold. Hence for the entire expression (1.5) to be finite, (1.6) must hold as well.

So we have seen, heuristically at least, that for the kind of polynomial $p$ under consideration here, if $U$ is a small enough neighborhood of the origin, then $\int_{U}|p|^{-\epsilon}<\infty$ exactly when $\epsilon<\frac{1}{N D(p)}$. Thus one might ask the question of how general this phenomenon is. Specifically, we ask the following two questions:

Question 1. For which smooth functions $f$, defined in a neighborhood of $(0,0)$ with $f(0,0)=0$, is it true that for any sufficiently small neighborhood $U$ of the origin we have $\int_{U}|f|^{-\epsilon}<\infty$ when $\epsilon<\frac{1}{N D(p)}$, and $\int_{U}|f|^{-\epsilon}=\infty$ when $\epsilon>\frac{1}{N D(p)}$ ? 
Question 2. When the condition of Question 1 holds, what can we say when $\epsilon=\frac{1}{N D(p)}$ ? In other words, in analogy to the one-dimensional case, is $\int_{U}|f|^{-\frac{1}{N D(p)}}$ infinite?

The purpose of this paper is to answer Questions 1 and 2. Somewhat different questions are addressed in $[\mathrm{P}]$ and $[\mathrm{PSSt}]$, where they seek coordinate systems for which $\frac{1}{N D(p)}$ gives the best $\epsilon$ modulo sharpness.

To give an indication of when Question 1 has a negative answer, suppose $f(x, y)=$ $(x-y)^{n}$ for some $n$. Then $\int_{U}|f|^{-\epsilon}=\infty$ when $\epsilon \geq \frac{1}{n}$, while $N D(f)=\frac{n}{2}$. Thus the condition of Question 1 does not hold. To see what is happening here, fix a positive number $d$, and observe that every $(x, y)$ in $\mathbf{R}^{2}$ can be written as $\left(x, c x^{d}\right)$ for some constant $c$. We consider the curves $B_{d}^{c}$ defined for a fixed $x_{0}$ by

$$
B_{d}^{c}=\left\{\left(x, c x^{d}\right): 0<x<x_{0}\right\} .
$$

Suppose $f(x, y)$ is any smooth function with possibly nonconvergent Taylor expansion $\sum_{m, n} f_{m, n} x^{m} y^{n}$. Let $e$ denote $\inf _{f_{m, n} \neq 0} m+n d$. Then on the curve $B_{d}^{c}$ we have that

$$
f(x, y)=f\left(x, c x^{d}\right)=\left(\sum_{m+n d=e} f_{m, n} c^{n}\right) x^{e}+o\left(x^{e}\right) .
$$

So, for example, in the case where $f(x, y)=(x-y)^{n}$, we have the expression $f(x, y)=(1-c)^{n} x^{e}$ along $B_{d}^{c}$. Now if we perform the integration $\int_{U}|f(x, y)|^{-\epsilon} d x d y$ in the $x$ and $c$ variables, if the function $g_{d}(c)=\sum_{m+n d=e} f_{m, n} c^{n}$ has a zero of high enough order in $c$, the integration in the $c$ variable will cause the integral to blow up on $x>0$ for smaller $\epsilon$ than it would if it had no zeroes at all (when Newton polygon dictates the situation). Similarly, if $\bar{g}_{d}(c)=\sum_{m+n d=e} f_{m, n}(-1)^{m} c^{n}$ has a zero of high enough order, the integration in $c$ will cause the integral over $x<0$ to blow up for small $\epsilon$.

What we will see is that the answer to Question 1 cannot only be expressed solely in terms of the maximum order of the zeroes of the $g_{d}(c)$ and $\bar{g}_{d}(c)$, but in fact at most one $d$ is relevant. If there is a bounded edge of $N(f)$ of some slope $-\frac{1}{\tilde{m}}$ intesecting the line $y=x$ in its interior, then $f(x, y)$ will satisfy the condition of Question 1 exactly when $g_{\tilde{m}}$ and $\bar{g}_{\tilde{m}}$ have zeroes of order at most $N D(f)$. If the line $y=x$ doesn't intersect the interior of a bounded edge of $N(f)$, then the condition of Question 1 is always satisfied. This as well as our answer to Question 2 are given by the following:

Main Theorem. Let $f(x, y)$ be a $C^{\infty}$ function defined in a neighborhood of the origin with $f(0,0)=0$ such that there is some $(a, b)$ with $\partial_{x}^{a} \partial_{y}^{b} f(0,0) \neq 0$. Denote the (possibly nonconvergent) Taylor expansion of $f(x, y)$ at $(0,0)$ by $\sum_{m, n} f_{m, n} x^{m} y^{n}$. For a neighborhood $U$ on which $f(x, y)$ is defined, we define

$$
I_{\epsilon}^{U}=\int_{U}|f(x, y)|^{-\epsilon} d x d y .
$$

In the case where the line $x=y$ intersects the interior of a bounded segment of $N(f)$, we let $-\frac{1}{\tilde{m}}$ denote the slope of that segment and $g_{\tilde{m}}, \bar{g}_{\tilde{m}}$ be as above.

If $U$ is a sufficiently small neighborhood of the origin (depending on $f$ ) we have:

1) If the line $y=x$ intersects the interior of a bounded segment of $N(f)$, and all zeroes of $g_{\tilde{m}}$ or $\bar{g}_{\tilde{m}}$ are of order at most $N D(f)$, then $I_{\epsilon}^{U}<\infty$ for $\epsilon<\frac{1}{N D(f)}$, and $I_{\epsilon}^{U}=\infty$ for $\epsilon \geq \frac{1}{N D(f)}$. 
2) If the line $y=x$ intersects the interior of a bounded segment of $N(f)$, and $g_{\tilde{m}}$ or $\bar{g}_{\tilde{m}}$ has a zero of order greater than $N D(f)$, then there exists a $\epsilon<\frac{1}{N D(f)}$ such that $I_{\epsilon}^{U}=\infty$.

3) If the line $y=x$ contains a vertex of $N(f)$, then $I_{\epsilon}^{U}<\infty$ for $\epsilon<\frac{1}{N D(f)}$, and $I_{\epsilon}^{U}=\infty$ for $\epsilon \geq \frac{1}{N D(f)}$.

4) If the line $y=x$ intersects the interior of an unbounded segment of $N(f)$, and $N(f)$ is neither the set $\{(x, y): x \geq 0, y \geq 1\}$ nor the set $\{(x, y): x \geq 1, y \geq 0\}$, then $I_{\epsilon}^{U}<\infty$ for $\epsilon<\frac{1}{N D(f)}$, and $I_{\epsilon}^{U}=\infty$ for $\epsilon>\frac{1}{N D(f)}$. In the case $\epsilon=\frac{1}{N D(f)}$, for any Newton polygon $N$ there are functions $f_{1}$ and $f_{2}$ with $N\left(f_{1}\right)=N\left(f_{2}\right)=N$ such that $I_{\epsilon}^{U} f_{1}=\infty$ while $I_{\epsilon}^{U} f_{2}<\infty$.

5) If $N(f)$ is either the set $\{(x, y): x \geq 0, y \geq 1\}$ or the set $\{(x, y): x \geq 1, y \geq$ $0\}$, then $I_{\epsilon}^{U}<\infty$ for $\epsilon<\frac{1}{N D(f)}$, and $I_{\epsilon}^{U}=\infty$ for $\epsilon \geq \frac{1}{N D(f)}$.

Thus we see Question 1 is answered in the negative only when $g_{\tilde{m}}$ or $\bar{g}_{\tilde{m}}$ has a zero of high enough order, and that Question 2 can only be answered in the negative if the line $y=x$ intersects the interior of an infinite edge of $N(f)$. To determine the best possible $\epsilon$ for which $I_{\epsilon}^{U}<\infty$ in case 2, one would have to look at finer resolutions of the zero set of $f(x, y)$ than one can with the use of $g_{\tilde{m}}$ or $\bar{g}_{\tilde{m}}$ alone.

\section{Some Lemmas}

In this section $f(x, y)$ is assumed to be a smooth finite-type function in a neighborhood of $(0,0)$ with Taylor expansion $\sum_{m, n} f_{m, n} x^{m} y^{n}$, such that $f(0,0)=0$. Expanding in $x$, for each $p$ we have that

$$
f(x, y)=\sum_{m=0}^{p-1} f_{m}(y) x^{m}+O\left(|x|^{p}\right) .
$$

Expanding each term of (2.1) in $y$, this becomes

$$
f(x, y)=\sum_{m<p, n<p} f_{m, n} x^{m} y^{n}+O\left(|x|^{p}+|y|^{p}\right) .
$$

The following lemmas are to be taken to hold in a sufficiently small neighborhood of $(0,0)$ depending only on $f$. The first lemma is a formalization of the heuristics of $(1.2)-(1.4)$.

Lemma 2.1. Suppose $(a, b)$ is a vertex of $N(f)$, between edges of slopes $-\frac{1}{m_{i}}$ and $-\frac{1}{m_{i+1}}$, where $m_{i}<m_{i+1}$. We allow the possibility of $m_{i}$ to be 0 and $m_{i+1}$ be $\infty$. Then there is an open set $U$ on which we have the estimates

$$
\frac{1}{2}\left|f_{a, b} x^{a} y^{b}\right|<f(x, y)<2\left|f_{a, b} x^{a} y^{b}\right| .
$$

$U$ may be taken as follows:

If $m_{i}>0$, and $m_{i+1}<\infty$, there are $\delta_{i}, N_{i}>0$ such that $U$ can be taken to be

$$
\left\{(x, y): N_{i}|x|^{m_{i+1}}<|y|<\delta_{i}|x|^{m_{i}}\right\} .
$$

If $m_{i}=0$, then in $(2.4), \delta_{i}|x|^{m_{i}}$ can be replaced by $C_{\rho}|x|^{\rho}$ for any $\rho$. If $m_{i+1}=\infty$, then in (2.4), $N_{i}|x|^{m_{i+1}}$ can be replaced by $C_{M}|x|^{M}$ for any $M$. If $m_{i}=0$ and $m_{i+1}=\infty$, both replacements can be made. 
In addition, if $a=0, U$ can be taken to be the whole set

$$
\left\{(x, y): N_{i}|x|^{m_{i+1}}<|y|\right\} .
$$

While if $b=0, U$ can be taken to be the whole set

$$
\left\{(x, y):|y|<\delta_{i}|x|^{m_{i}}\right\} .
$$

Proof. We restrict attention to the upper right quadrant as the other three are done the same way. First we suppose that $m_{i}>0$ and $m_{i+1}<\infty$. Let $U$ be as in the statement of the lemma. Write (2.2) in the form

$$
\begin{gathered}
\left(\frac{1}{2} f_{a, b} x^{a} y^{b}+\sum_{m \leq a, b<n<p} f_{m, n} x^{m} y^{n}\right)+\left(\frac{1}{2} f_{a, b} x^{a} y^{b}+\sum_{n \leq b, a<m<p} f_{m, n} x^{m} y^{n}\right) \\
+\sum_{a<m<p, b<n<p} f_{m, n} x^{m} y^{n}+O\left(|x|^{p}+|y|^{p}\right) \\
=S_{1}+S_{2}+S_{3}+O\left(|x|^{p}+|y|^{p}\right) .
\end{gathered}
$$

In $S_{1}$, we change variables $y=x^{m_{i}} y^{\prime}$. The condition $|y|<\delta_{i}|x|^{m_{i}}$ becomes $\left|y^{\prime}\right|<\delta_{i}$, and $S_{1}$ can be written as

$$
x^{a+m_{i} b}\left[\frac{1}{2} f_{a, b}\left(y^{\prime}\right)^{b}+O\left(\left(y^{\prime}\right)^{b+1}\right)\right] .
$$

Here $\eta=\min \left(m_{i}, 1\right)>0$. Thus if $\delta_{i}$ is small enough to take care of the $O\left(\left(y^{\prime}\right)^{b+1}\right)$ and $O\left(x^{a+m_{i} b+\eta}\left(y^{\prime}\right)^{b+1}\right)$ terms, we have that

$$
\left|S_{1}-\frac{1}{2} f_{a, b} x^{a} y^{b}\right|<\frac{1}{8}\left|f_{a, b} x^{a} y^{b}\right| .
$$

Analogously, reversing the roles of the $x$ and $y$ axes, if $N_{i}$ is sufficiently large, we have

$$
\left|S_{2}-\frac{1}{2} f_{a, b} x^{a} y^{b}\right|<\frac{1}{8}\left|f_{a, b} x^{a} y^{b}\right| .
$$

As for $S_{3}$, each $f_{m, n} x^{m} y^{n}$ appearing there has $m>a$ and $n>b$, so assuming $x$ and $y$ are sufficiently small, which we may, we have

$$
\left|S_{3}\right|<\frac{1}{8}\left|f_{a, b} x^{a} y^{b}\right| .
$$

Finally, since we assume $m_{i}>0$ and $m_{i+1}<\infty$, there exists a $\xi>0$ and constants $C, C^{\prime}$ such that $C|x|^{\xi}<|y|<C^{\prime}|x|^{\frac{1}{\xi}}$ in $U$. The left inequality gives $|x|<C^{\prime \prime}|x|^{a \mu}|y|^{b \mu}$ and the right inequality gives $|y|<C^{\prime \prime \prime}|x|^{a \mu}|y|^{b \mu}$ for some small $\mu>0$. Thus if $p$ is sufficiently large, we have for some constant $C$ that

$$
|x|^{p}+|y|^{p}<C|x|^{2 a}|y|^{2 b} .
$$

So when $|x|$ and $|y|$ are sufficiently small, if $S_{4}$ denotes the $O\left(|x|^{p}+|y|^{p}\right)$ error term in $(2.7)$, we must have

$$
\left|S_{4}\right|<\frac{1}{8}\left|f_{a, b} x^{a} y^{b}\right| .
$$

Combining (2.9) - (2.12) gives us (2.3) in the case that $m_{i} \neq 0$ and $m_{i+1} \neq \infty$.

Suppose now $m_{i}=0$, and we replace the condition $|y|<\delta_{i}|x|^{m_{i}}$ by the condition that $|y|<C_{\rho}|x|^{\rho}$ for every $\rho>0$. Then (2.9) - (2.12) follow very much like above. For now $S_{1}$ is just $\frac{1}{2} f_{a, b} x^{a} y^{b}$ and (2.9) is automatic. (2.10) and (2.11) did not use the condition $|y|<\delta_{i}|x|^{m_{i}}$ and therefore still hold. (2.12) used $|y|<\delta_{i}|x|^{m_{i}}$ by 
virtue of the fact that $|y|<C^{\prime}|x|^{\frac{1}{\xi}}$ for some $\xi>0$, which still holds in the present case. Thus we have (2.12) as well. We conclude that (2.3) holds when $m_{i}=0$. The case where $m_{i+1}=\infty$ is handled similarly with the roles of the $x$ and $y$ axes reversed. If $m_{i}=0$ and $m_{i+1}=\infty,(2.9)$ and (2.10) are immediate, and (2.11), (2.12) are as in the previous paragraphs.

Finally we consider the statement of Lemma 2.1 regarding the situation when $a$ or $b$ is zero. (They can't both be zero since we are assuming $f(0,0)=0$.) For variation, we consider the case where $b=0$; the case $a=0$ is done the analogous way. We assume now only an inequality of the form $|y|<\delta_{i}|x|^{m_{i}}$, and our goal once again is to prove $(2.9)-(2.12)$. Again we restrict to the upper right quadrant. Estimate (2.9) follows exactly as before; its proof uses that $|y|<\delta_{i}|x|^{m_{i}}$ but not that $|y|>C_{M}|x|^{M}$. $S_{2}$ is equal to $\frac{1}{2} f_{a, b} x^{a} y^{b}$ and (2.10) follows. (2.11) is done exactly as before. Finally, since $b=0, f_{a, b} x^{a} y^{b}=f_{a, b} x^{a}$ and thus if $p$ is sufficiently large, we have

$$
S_{4}=O\left(|x|^{p}+|y|^{p}\right)=O\left(|x|^{p}+|x|^{p m_{i}}\right)=O\left(|x|^{\min \left(p, p m_{i}\right)}\right)=O\left(|x|^{a+1}\right) .
$$

Hence if $|x|$ is sufficiently small, we have $S_{4}<\frac{1}{8} f_{a, b}|x|^{a}=\frac{1}{8} f_{a, b}|x|^{a}|y|^{b}$, and (2.12) follows.

Having exhausted all cases, we have completed the proof of Lemma 2.1.

The next lemma formalizes the heuristics of (1.7) and the subsequent discussion.

Lemma 2.2. Suppose $N(f)$ has a bounded face of slope $-\frac{1}{m_{i}}$. Let e be the minimal value of $m+m_{i} n$ over all $(m, n)$ such that $f_{m, n}$ is nonzero. As before let $g_{m_{i}}(c)$ denote the polynomial $\sum_{m+m_{i} n=e} f_{m, n} c^{n}$. For $s>r>0$, define

$$
V_{r}^{s}=\left\{(x, y): x>0, r x^{m_{i}} \leq y \leq s x^{m_{i}}\right\} .
$$

1) If $g_{m_{i}}(c)$ has no zeroes for $r \leq c \leq s$, then there are $\delta, N>0$ such that

$$
\delta x^{e}<|f(x, y)|<N x^{e} \quad\left((x, y) \in V_{r}^{s}\right) .
$$

2) If $g_{m_{i}}(c)$ has exactly one zero $c=c_{0}$ in $[r, s]$, of order $q$, then there exists $\mu>0$ and constants $C, \delta$, and $N$ such that if $(x, y)$ is in $V_{r}^{s}$ and $\left|y-c_{0} x^{m_{i}}\right|>C|x|^{m_{i}+\mu}$, we have

$$
\delta x^{e-q m_{i}}\left(y-c_{0} x^{m_{i}}\right)^{q}<|f(x, y)|<N x^{e-q m_{i}}\left(y-c_{0} x^{m_{i}}\right)^{q} .
$$

The analogous statements hold for $x<0$, with $g_{m_{i}}(c)$ replaced by $\bar{g}_{m_{i}}(c)$.

Proof. Do the variable change $y=x^{m_{i}} y^{\prime}$. Then in the new coordinates we have

$$
V_{r}^{s}=\left\{\left(x, y^{\prime}\right): x>0, r \leq y^{\prime} \leq s\right\} .
$$

For a small $\eta>0$ we have

$$
f\left(x, x^{m_{i}} y^{\prime}\right)=g_{m_{i}}\left(y^{\prime}\right) x^{e}+O\left(x^{e+\eta}\right) .
$$

Suppose $g_{m_{i}}$ has no zeroes on $[r, s]$. We may define $\delta$ and $N$ such that on $[r, s]$ we have $\frac{N}{2}>\left|g_{m_{i}}\right|>2 \delta$. Assuming $x$ is sufficiently small such that the $O\left(x^{e+\eta}\right)$ term is of absolute value less than $\delta x^{e}$, we have

$$
\delta x^{e}<\left|f\left(x, x^{m_{i}} y^{\prime}\right)\right|<N x^{e} .
$$

Changing variables back from $y^{\prime}$ to $y$ gives (2.15) and we are done with part 1) of the lemma. 
Moving now to part 2), suppose $g_{m_{i}}$ has a zero of order $q$ at $c_{0}$, and no other on the interval $[r, s]$. Thus for some $M, \nu>0$ we have

$$
M\left(y^{\prime}-c_{0}\right)^{q}>\left|g_{m_{i}}\left(y^{\prime}\right)\right|>\nu\left(y^{\prime}-c_{0}\right)^{q} \quad\left(y^{\prime} \in[r, s]\right) .
$$

Now if $\left|y^{\prime}-c_{0}\right|>x^{\frac{\eta}{2 q}}, \eta$ is as in (2.17), the term $g_{m_{i}}\left(y^{\prime}\right) x^{e}$ satisfies

$$
\left|g_{m_{i}}\left(y^{\prime}\right) x^{e}\right|>\nu\left(y^{\prime}-c_{0}\right)^{q} x^{e}>\nu x^{e+\frac{\eta}{2}} .
$$

So for $x$ sufficiently small the $O\left(x^{e+\eta}\right)$ term in $(2.17)$ is of absolute value at most half that of $g_{m_{i}}\left(y^{\prime}\right) x^{e}$. Hence if $x$ is sufficiently small and $\left|y^{\prime}-c_{0}\right|>x^{\frac{\eta}{2 q}}$, in view of (2.17) and (2.18), we have

$$
\frac{\nu}{2} x^{e}\left(y^{\prime}-c_{0}\right)^{q}<\left|f\left(x, x^{m_{i}} y^{\prime}\right)\right|<2 M x^{e}\left(y^{\prime}-c_{0}\right)^{q} .
$$

Translating (2.19) back from $y^{\prime}$ to $y$ variables gives (2.16) and the proof of Lemma 2.2 is complete.

The next lemma is a Van Der Corput-type lemma we need for the arguments of Section 3.

Lemma 2.3. Suppose $g(y)$ is a $k$-times differentiable function on an interval $I$, with $\left|g^{(k)}\right|>\eta$. Then if $\epsilon<\frac{1}{k}$, we have

$$
\int_{I}|g(y)|^{-\epsilon} d y<C \eta^{-\epsilon}|I|^{1-k \epsilon} .
$$

In other words,

$$
\int_{I}|g(y)|^{-\epsilon} d y<C^{\prime} \int_{0}^{|I|}\left(\eta z^{k}\right)^{-\epsilon} d z .
$$

Here $C$ and $C^{\prime}$ depend on $\epsilon$ and $k$.

Proof. Let $F(\lambda)$ be the distribution function of $|g|$. By one version of the Van der Corput Lemma (see [C]) we have

$$
F(\lambda)<C \frac{\lambda^{\frac{1}{k}}}{\eta^{\frac{1}{k}}}
$$

In particular, we have that $\lim _{\lambda \rightarrow 0} \frac{F(\lambda)}{\lambda^{\epsilon}}=0$. Hence we may integrate by parts in the right-hand side of the following equation:

$$
\int_{I}|g(y)|^{-\epsilon} d y=\int \lambda^{-\epsilon} d F(\lambda) .
$$

The result is

$$
\int_{I}|g(y)|^{-\epsilon} d y=\epsilon \int_{0}^{\infty} \lambda^{-1-\epsilon} F(\lambda) d \lambda .
$$

We break (2.23) into two parts, from 0 to $\lambda_{0}$ and from $\lambda_{0}$ to infinity, for a $\lambda_{0}$ to be specified shortly. On the left portion, we use (2.22), while on the right portion we use that $F(\lambda) \leq|I|$. Naturally, $\lambda_{0}$ is chosen such that $|I|=\frac{\lambda_{0}^{\frac{1}{k}}}{\eta^{\frac{1}{k}}}$, or in other words, we choose

$$
\lambda_{0}=\eta|I|^{k}
$$


Having chosen this $\lambda_{0},(2.23)$ becomes

$$
\int_{I}|g(y)|^{-\epsilon} d y<C \eta^{-\epsilon}|I|^{1-k \epsilon} .
$$

Thus the proof of Lemma 2.3 is complete.

\section{Proof of the Main Theorem}

We are now in a position to prove the Main Theorem. We prove each of the five statements in succession.

Statement 1): Suppose the line $y=x$ intersects $N(f)$ in the interior of a bounded segment with vertices $(a, b)$ and $\left(a^{\prime}, b^{\prime}\right)$, where $a>a^{\prime}$ and $b<b^{\prime}$. Denote the slope $\frac{b^{\prime}-b}{a^{\prime}-a}$ of this segment by $-\frac{1}{\tilde{m}}$ as before. Then $N\left(\partial_{y}^{b} f\right)$ has a vertex at $(a, 0)$, so by Lemma 2.1 there is a $\mu>0$ such that $\left|\partial_{y}^{b} f\right|>C|x|^{a}$ on the set $U_{1}=\{(x, y):|y|<$ $\left.\mu|x|^{\tilde{m}}\right\}$. For fixed $x$, denote by $U_{1}^{y}$ the set $\left\{(x, y): y \in U_{1}\right\}$. By Lemma 2.3, if $\epsilon<\frac{1}{N D(f)}<\frac{1}{b}$ we have

$$
\int_{U_{1}^{x}}|f(x, y)|^{-\epsilon} d y<C|x|^{-a \epsilon+\tilde{m}(1-b \epsilon)} .
$$

Observe that $-a \epsilon+\tilde{m}(1-b \epsilon)>-1$ because $\epsilon<N D(f)=\frac{1+\tilde{m}}{a+\tilde{m} b}$, so that we have

$$
\int_{U_{1}}|f(x, y)|^{-\epsilon} d x d y<\infty
$$

Similarly, reversing the roles of the $x$ and $y$ variables, there is a set $U_{2}$ of the form $\left\{(x, y):|y|>M|x|^{\tilde{m}}\right\}$ such that

$$
\int_{U_{2}}|f(x, y)|^{-\epsilon} d x d y<\infty
$$

Hence it remains to consider the set of points $(x, y)$ such that $\mu|x|^{\tilde{m}}<|y|<M|x|^{\tilde{m}}$. We will restrict consideration to the points where $x>0$, as the case $x<0$ is analogous with $g_{\tilde{m}}$ replaced by $g_{\tilde{m}}^{\prime}$. We define

$$
U_{1}=\left\{(x, y): x>0, \mu x^{\tilde{m}}<|y|<M x^{\tilde{m}}\right\} .
$$

Let $c_{0}, \ldots, c_{l}$ be the zeroes of $g_{\tilde{m}}$ on $[\mu, M]$. Enlarging $[\mu, M]$ if necessary, we may assume neither $\mu$ nor $M$ is a zero of $g_{\tilde{m}}$. For each $i$, let $r_{i}<r_{i}^{\prime}$ be such that $c_{i}$ is the unique zero of $g_{\tilde{m}}$ on $\left[r_{i}, r_{i}^{\prime}\right]$. Define the regions $V_{i}$ by

$$
V_{i}=\left\{(x, y): x>0, r_{i} x^{\tilde{m}}<y<r_{i}^{\prime} x^{\tilde{m}}\right\} .
$$

We also divide the interior of $U_{1}-\bigcup_{i} V_{i}$ into regions $W_{i}$, where $W_{i}$ is of the same form:

$$
W_{i}=\left\{(x, y): x>0, \quad s_{i} x^{\tilde{m}}<y<s_{i}^{\prime} x^{\tilde{m}}\right\} .
$$

By Lemma 2.2 part 1), we can find $\delta^{i}, N^{i}>0$ such that on $W_{i}$ we have

$$
\delta^{i}|x|^{a+\tilde{m} b}<|f(x, y)|<N^{i}|x|^{a+\tilde{m} b} .
$$

Denoting by $W_{i}^{x}$ the set $\left\{(x, y): y \in W_{i}\right\}$, we thus have

$$
C_{1}|x|^{-\epsilon a-\epsilon \tilde{m} b+\tilde{m}}<\int_{W_{i}^{x}}|f(x, y)|^{-\epsilon} d y<C_{2}|x|^{-\epsilon a-\epsilon \tilde{m} b+\tilde{m}} .
$$

Hence $|f(x, y)|^{-\epsilon}$ is integrable on $W_{i}$ because the exponent $-\epsilon a-\epsilon \tilde{m} b+\tilde{m}$ is greater than -1 ; this again holds because we are assuming $\epsilon<\frac{1}{N D(f)}$. In addition, (3.5) 
implies that $\int_{W_{i}}|f(x, y)|^{-\frac{1}{N D(f)}}=\infty$. As a result, $I_{t}^{U}=\infty$ for $t \geq \frac{1}{N D(f)}$, proving one part of statement 1) of the Main Theorem.

We now focus our attention on the sets $V_{i}$. Let $q_{i}$ denote the order of the zero of $g_{\tilde{m}}$ at $c_{i} . f(x, y)$ can be Taylor expanded as

$$
f(x, y)=\sum_{m+\tilde{m} n=e} f_{m, n} x^{m} y^{n}+\sum_{m+\tilde{m} n>e} f_{m, n<n} x^{m} y^{n}+O\left(|x|^{p}+|y|^{p}\right) .
$$

Correspondingly, $\partial_{y}^{q_{i}} f(x, y)$ can be Taylor expanded as

$$
\partial_{y}^{q_{i}} f(x, y)=\sum_{m+\tilde{m} n=e, n \geq q_{i}} n(n-1) \ldots\left(n-q_{i}+1\right) f_{m, n} x^{m} y^{n-q_{i}}
$$

$$
+\sum_{m+\tilde{m} n>e,} \sum_{m<p, q_{i} \leq n<p} n(n-1) \ldots\left(n-q_{i}+1\right) f_{m, n} x^{m} y^{n-q_{i}}+O\left(|x|^{p}+|y|^{p-q_{i}}\right) .
$$

By definition of $q_{i}$, the function $\sum_{m+\tilde{m} n=e} n \geq q_{i} n(n-1) \ldots\left(n-q_{i}+1\right) f_{m, n} y^{n-q_{i}}$ has no zeroes on the interval $\left[r_{i}, r_{i}^{\prime}\right]$. As a result, we may apply Lemma 2.2 part 1) to conclude that on $V_{i}$ we have

$$
C_{1} x^{a+\tilde{m}\left(b-q_{i}\right)}<\left|\partial_{y}^{q_{i}} f(x, y)\right|<C_{2} x^{a+\tilde{m}\left(b-q_{i}\right)} .
$$

Define $V_{i}^{x}=\left\{(x, y): y \in V_{i}\right\}$. Then by Lemma 2.3, which we may apply since $\epsilon<\frac{1}{N D(f)} \leq \frac{1}{q_{i}}$ (here is where we use the condition $q_{i} \leq N D(f)$ ), we have

$$
\int_{V_{i}^{x}}|f(x, y)|^{-\epsilon} d y<C_{3} x^{-\epsilon a-\epsilon \tilde{m}\left(b-q_{i}\right)+\left(1-q_{i} \epsilon\right) \tilde{m}} .
$$

In order for the expression in (3.8) to be integrable, we must have the condition

$$
-\epsilon a-\epsilon \tilde{m}\left(b-q_{i}\right)+\left(1-q_{i} \epsilon\right) \tilde{m}>-1 .
$$

This translates once again into the condition $\epsilon<\frac{1+m}{a+m b}=N D(f)$, which is what we are assuming. Thus we are done with assertion 1) of the Main Theorem.

Moving on to statement 2) of the Main Theorem: Without loss of generality, we assume $g_{\tilde{m}}$ has a zero $c_{0}$ of order $q_{0}>N D(f)$ as the case where $\bar{g}_{\tilde{m}}$ has a zero of order greater than $N D(f)$ is done in much the same way. Let $\left[r, r^{\prime}\right]$ be an interval with $c_{0}$ in its interior, but which contains no other zeroes of $g_{\tilde{m}}$. Then we can apply Lemma 2.2 part 2) to conclude that for some $\mu>0$, some constants $C, C_{1}$, and $C_{2}$ there is a set

$$
V=\left\{(x, y): c_{0} x^{\tilde{m}}+C x^{\tilde{m}+\mu}<y<r^{\prime} x^{\tilde{m}}\right\}
$$

such that on $V$ we have (where $(a, b)$ is as in the proof of part 1 ) of this theorem)

$$
C_{1} x^{a+\tilde{m}\left(b-q_{0}\right)}\left(y-c_{0} x^{\tilde{m}}\right)^{q_{0}}<|f(x, y)|<C_{2} x^{a+\tilde{m}\left(b-q_{0}\right)}\left(y-c_{0} x^{\tilde{m}}\right)^{q_{0}} .
$$

Define $V^{x}=\{y:(x, y) \in V\}$. Then if $\frac{1}{q_{0}}<\epsilon<\frac{1}{N D(f)}$, by (3.9), we have

$$
\begin{aligned}
C_{3} x^{-\epsilon\left(a+\tilde{m}\left(b-q_{0}\right)\right)+\left(1-\epsilon q_{0}\right)(\tilde{m}+\mu)} & \\
& <\int_{V^{x}}|f(x, y)|^{-\epsilon} d y<C_{4} x^{-\epsilon\left(a+\tilde{m}\left(b-q_{0}\right)\right)+\left(1-\epsilon q_{0}\right)(\tilde{m}+\mu)} .
\end{aligned}
$$

The exponent of $x$ appearing in (3.10) reduces to

$$
-\epsilon a-\epsilon \tilde{m} b+\tilde{m}+\mu\left(1-\epsilon q_{0}\right) .
$$


If we replaced $\epsilon$ by $\frac{1}{N D(f)}$, then $-\epsilon a-\epsilon \tilde{m} b+\tilde{m}$ would equal -1 by the definition of $N D(f)$. Since $1-\frac{q_{0}}{N D(f)}<0$, replacing $\epsilon$ by $\frac{1}{N D(f)}$ in (3.11) gives a quantity less than -1 . Hence by contiuity there exists an $\epsilon$ with $\frac{1}{q_{0}}<\epsilon<\frac{1}{N D(f)}$ for which (3.11) is less than -1 . In this case, by (3.10) we must have

$$
\int_{V}|f(x, y)|^{-\epsilon} d x d y=\infty .
$$

This completes the proof of part 2) of the Main Theorem.

Continuing to part 3), we suppose now $N(f)$ has a vertex $(a, a)$ for some $a>0$, between sides of slopes $-\frac{1}{m}$ and $-\frac{1}{m^{\prime \prime}}$ for $m<m^{\prime}$. $m$ and $m^{\prime}$ can be 0 and infinity respectively. Let $m^{\prime \prime}$ satisfy $m<m^{\prime \prime}<m^{\prime}$. We divide our small neighborhood $U$ of the origin into two parts $U_{1}$ and $U_{2}$, where

$$
\begin{aligned}
& U_{1}=\left\{(x, y) \in U:|y|<|x|^{m^{\prime \prime}}\right\}, \\
& U_{2}=\left\{(x, y) \in U:|y| \geq|x|^{m^{\prime \prime}}\right\} .
\end{aligned}
$$

By Lemma 3.1, if $U$ is small enough, then we have

$$
\begin{aligned}
C|x|^{a}<\left|\partial_{y}^{a} f(x, y)\right|<C^{\prime}|x|^{a} & & \left((x, y) \in U_{1}\right), \\
C|y|^{a}<\left|\partial_{x}^{a} f(x, y)\right|<C^{\prime}|y|^{a} & & \left((x, y) \in U_{2}\right) .
\end{aligned}
$$

Hence by Lemma 3.3, if we denote the set $\left\{(x, y): y \in U_{1}\right\}$ by $U_{1}^{x}$, and we denote the set $\left\{(x, y): x \in U_{2}\right\}$ by $U_{2}^{y}$, we have for any $\epsilon<\frac{1}{a}$,

$$
\begin{aligned}
& C_{1}|x|^{-a \epsilon+(1-a \epsilon) m^{\prime \prime}}<\int_{U_{1}^{x}}|f(x, y)|^{-\epsilon} d y<C_{2}|x|^{-a \epsilon+(1-a \epsilon) m^{\prime \prime}}, \\
& C_{3}|y|^{-a \epsilon+\frac{(1-a \epsilon)}{m^{\prime \prime}}}<\int_{U_{2}^{y}}|f(x, y)|^{-\epsilon} d x<C_{4}|y|^{-a \epsilon+\frac{1-a \epsilon}{m^{\prime \prime}}} .
\end{aligned}
$$

Since $0<a \epsilon<1$, the exponents appearing in (3.14a) and (3.14b) are both greater than -1 , and we conclude that

$$
\int_{U}|f(x, y)|^{-\epsilon} d x d y=\int_{U_{1}}|f(x, y)|^{-\epsilon} d x d y+\int_{U_{2}}|f(x, y)|^{-\epsilon} d x d y<\infty .
$$

On the other hand, suppose $\epsilon=\frac{1}{a}$. Let $n, n^{\prime}$ satisfy $m<n<n^{\prime}<m^{\prime}$. Define

$$
U_{3}=\left\{(x, y) \in U:|x|^{n^{\prime}}<|y|<|x|^{n}\right\} .
$$

Then by Lemma 2.1, if $U$ is sufficiently small, for each $(x, y)$ in $U_{3}$ we must have

$$
C_{5}|x|^{a}|y|^{a}<|f(x, y)|<C_{6}|x|^{a}|y|^{a} .
$$

As a result, since $a=\frac{1}{\epsilon}$, we conclude that

$$
\int_{U_{3}}|f(x, y)|^{-\frac{1}{\epsilon}}>C_{5} \int_{U_{3}}|x|^{-1}|y|^{-1}=\infty .
$$

This completes the proof of part 3) of the Main Theorem.

Proceeding to part 4), suppose the line $y=x$ intersects $N(f)$ in the interior of a horizontal line $y=b$. (The case of a vertical line is treated in an analogous fashion.) Let $(a, b)$ be the corresponding vertex of $N(f)$, and denote by $-\frac{1}{m}$ the slope of the nonhorizontal edge of $N(f)$ containing $(a, b)$. As usual $m$ is allowed to 
be zero. In the case that $m \neq 0$, by Lemma 2.1 there is a $\delta>0$ such that if in a small neighborhood $U$ of the origin we define

$$
V=\left\{(x, y) \in U:|y|<\delta|x|^{m}\right\} .
$$

Then for any $(x, y)$ in $V$ we have

$$
C_{1}|x|^{a}<\left|\partial_{y}^{b} f(x, y)\right|<C_{2}|x|^{a} .
$$

Similarly, if $(x, y) \in U-V$, then

$$
C_{3}|y|^{b}<\left|\partial_{x}^{a} f(x, y)\right|<C_{4}|y|^{b} .
$$

If $m=0$, then we just let $V$ be $\{(x, y):|y|<|x|\}$ and (3.16a) - (3.16b) still hold, again using Lemma 2.1.

By Lemma 2.3, if $\epsilon<\frac{1}{b}$, we have

$$
\begin{gathered}
C_{4}|x|^{-a \epsilon+(1-b \epsilon) m}<\int_{V_{x}}|f(x, y)|^{-\epsilon} d y<C_{5}|x|^{-a \epsilon+(1-b \epsilon) m}, \\
C_{6}|y|^{-b \epsilon+\frac{1-a \epsilon}{m}}<\int_{(U-V)_{y}}|f(x, y)|^{-\epsilon} d x<C_{5}|y|^{-b \epsilon+\frac{1-b \epsilon}{m}} .
\end{gathered}
$$

Since $\epsilon<\frac{1}{b}<\frac{1}{a}$, the exponents in (3.17a) and (3.17b) are both greater than -1 , and we conclude that whenever $\epsilon<\frac{1}{b}$ we have

$$
\int_{U}|f(x, y)|^{-\epsilon}<\infty
$$

Suppose now $\epsilon>\frac{1}{b}$. We write $\epsilon$ in the suggestive form $\frac{-1-\nu}{b}$ for $\nu>0$. Let $M$ be large enough so that $\frac{-a(1+\nu)}{b}-M \nu<-1$. By Lemma 2.1, there are $C_{M}, \delta^{\prime}>0$ such that on the set $W=\left\{(x, y): C_{M}|x|^{M}<|y|<\delta^{\prime}|x|^{m}\right\}$ we have a relation of the form

$$
C_{1}|x|^{a}|y|^{b}<|f(x, y)|<C_{2}|x|^{a}|y|^{b} .
$$

Therefore

$$
C_{1}|x|^{-a \epsilon+(1-b \epsilon) M}<\int_{W_{x}}|f(x, y)|^{-\epsilon} d y<C_{2}|x|^{-a \epsilon+(1-b \epsilon) M} .
$$

$M$ was defined exactly so that the exponent of $x$ in (3.19) is less than -1 , so we conclude that

$$
\int_{U}|f(x, y)|^{-\epsilon}=\infty
$$

This holds whenever $\epsilon>\frac{1}{b}$. Finally we consider the case when $\epsilon=\frac{1}{b}$. Let $N$ be any possible Newton polygon corresponding to the case at hand. Again we denote the lowest vertex of $N$ by $(a, b)$ and the slope of the segment to the left of it by $m$. Denote the other vertices of $N$ by $\left(a_{0}, b_{0}\right), \ldots,\left(a_{l}, b_{l}\right)$. We define

$$
p(x, y)=x^{a} y^{b}+\sum_{i=0}^{l} x^{a_{i}} y^{b_{i}} .
$$

Observe that the Newton polygon of $p$ is given by $N$. In the case that $m=0$, $p(x, y)=x^{a} y^{b}$ and $\int|p(x, y)|^{-\frac{1}{b}} d x d y$ is clearly infinite. For $m \neq 0$ we do the variable change $(x, y)=\left(x, x^{m} y^{\prime}\right)$, and we have for some $\eta>0$,

$$
p\left(x, x^{m} y^{\prime}\right)=x^{a+m b}\left(y^{\prime}\right)^{b}+O\left(x^{a+m b+\eta}\left(y^{\prime}\right)^{b+1}\right) .
$$


Hence if $\left|y^{\prime}\right|$ is sufficiently small, then $p\left(x, x^{m} y^{\prime}\right)$ is dominated by the first term of (3.20). Changing back to the original coordinates, we have that $|f(x, y)|$ is comparable to $|x|^{a}|y|^{b}$, so long as $|y|<\delta|x|^{m}$, for appropriately small $\delta$. Since the $-\frac{1}{b}$ power of $|x|^{a}|y|^{b}$ is not integrable over the set $|y|<\delta|x|^{m}$, the same must be true for $p(x, y)$. We conclude that

$$
\int_{U}|p(x, y)|^{-\frac{1}{b}}=\infty .
$$

On the other hand, suppose we change $p$ to

$$
f(x, y)=x^{a} y^{b-2} e^{|x|^{-\frac{1}{2 b}}}+x^{a} y^{b}+\sum_{i=0}^{l} x^{a_{i}} y^{b_{i}} .
$$

(We must have $b \geq 2$; otherwise we would be in case 5) of the Main Theorem.) Notice that the Newton polygon of $f(x, y)$ is once again $N$. However, we will see that this time $\int_{U}|f(x, y)|^{-\frac{1}{b}} d x d y$ is finite. Let $V$ again be as defined above (3.16a). Then (3.16b) and therefore (3.17b) hold exactly as before, and again since $a<b$ the exponent in $(3.17 \mathrm{~b})$ is greater than -1 . We thus again have

$$
\int_{U-V}|f(x, y)|^{-\frac{1}{b}} d x d y<\infty .
$$

Also, by shrinking the $\delta$ in $V$ 's definition if necessary, as before we can assume that on $V$ we have

$$
\left|x^{a} y^{b}\right|>\sum_{i=0}^{l}\left|x^{a_{i}} y^{b_{i}}\right| .
$$

Therefore on $V$, the magnitude of $f(x, y)$ is comparable to that of the sum of the first two terms of (3.21). These two terms are of the same sign. The first is of greater absolute value when $e^{|x|^{-\frac{1}{2 b}}}>y^{2}$, and the second is of greater absolute value when $e^{|x|^{-\frac{1}{2 b}}}<y^{2}$. As a result, the integral of the $-\frac{1}{b}$ th power of (3.21) in the $y$ direction on $V$ is finite since the second term dominates when $y$ is small. In fact, we have

$$
\begin{aligned}
& \int_{V}|f(x, y)|^{-\frac{1}{b}} d x d y \\
& \quad<C \int_{\left\{y \in V, y^{2}>e^{\left.|x|^{-\frac{1}{2 b}}\right\}}\right.}\left(|x|^{a}|y|^{b}\right)^{-\frac{1}{b}} d x d y<C \int|x|^{-\frac{a}{b}-\frac{1}{2 b}} d x .
\end{aligned}
$$

Since $a$ is an integer less than $b$, we have that $-\frac{a}{b}-\frac{1}{2 b}>-1$ and the right-hand term of (3.24) is integrable. Hence we have

$$
\int_{U}|f(x, y)|^{-\frac{1}{b}} d x d y=\int_{V}|f(x, y)|^{-\frac{1}{b}} d x d y+\int_{U-V}|f(x, y)|^{-\frac{1}{b}} d x d y<\infty .
$$

This completes the proof of part 4) of the Main Theorem.

Finally, we consider part 5) of the Main Theorem. We look only at the case where $N(f)=\{(x, y): x \geq 0, y \geq 1\}$ as the other case is done the same way. In this case $f(0,0)=0$ and $\partial_{y} f(0,0) \neq 0$, so by the implicit function theorem there is a neighborhood $U$ of $(0,0)$ and coordinates $\left(x^{\prime}, y^{\prime}\right)$ on $U$ such that in the new coordinates $f\left(x^{\prime}, y^{\prime}\right)=y^{\prime}$. Conclusion 5) of the Main Theorem immediately follows, and we are done. 


\section{REFERENCES}

[CCW] A. Carbery, M. Christ, J. Wright, Multidimensional van der Corput and sublevel set estimates, J. Amer. Math. Soc. 12 (1999), 981-1015. MR2000h:42010

[C] M. Christ, Hilbert transforms along curves. I. Nilpotent groups, Annals of Mathematics (2) 122 (1985), no.3, 575-596. MR87f:42039a

[PSSt] D. H. Phong, E. M. Stein, J. Sturm, On the growth and stability of real-analytic functions, American J. Math. 121 (1999), 519-554. MR2002a:58025

[P] M. Pramanik, Convergence of two-dimensional weighted integrals, Trans. Amer. Math. Soc. 354 (2002), 1651-1665. MR2003a:41033

[V] A. N. Varchenko, Newton polyhedra and estimates of oscillatory integrals, Functional Anal. Appl. 18 (1976), no. 3, 175-196.

Department of Mathematics, Massachusetts Institute of Technology, 77 MassachuSetts Avenue, Cambridge, Massachusetts 02139 